ISSN 1561-2430 (Print)

ISSN 2524-2415 (Online)

УДК 539.12

https://doi.org/10.29235/1561-2430-2019-55-1-97-109

Поступила в редакцию 11.09.2018

Received 11.09.2018

\author{
Д. В. Шёлковый ${ }^{1}$, Д. Н. Григорьев ${ }^{2,3,4}$, Л. Б. Эпштейн ${ }^{2,3,4}$, Ю. В. Юдин ${ }^{2,4}$ \\ ${ }^{1}$ Институт физики им. Б. И. Степанова Национальной академии наук Беларуси, Минск, Беларусь \\ ${ }^{2}$ Институт ядерной физики им. Г. И. Будкера СО РАН, Новосибирск, Россия \\ ${ }^{3}$ Новосибирский государственный технический университет, Новосибирск, Россия \\ ${ }^{4}$ Новосибирский государственный университет, Новосибирск, Россия

\section{СИСТЕМА ПРЕДВАРИТЕЛЬНОГО ОТБОРА СОБЫТИЙ ЭЛЕКТРОМАГНИТНОГО КАЛОРИМЕТРА ЭКСПЕРИМЕНТА СОМЕТ}

Аннотация. Для предварительного отбора событий по сигналам электромагнитного калориметра эксперимента COMET был разработан алгоритм обработки сигналов, позволяющий измерять в режиме реального времени энергии частиц, попадающих в калориметр. В данной работе описан процесс и результаты оптимизации этого алгоритма, проведенной с помощью моделирования методом Монте-Карло. Определены энергетические пороги на триггерные ячейки, позволяющие выделить сигнальное событие - электрон с импульсом $105 \mathrm{MэB/c,} \mathrm{и} \mathrm{значительно} \mathrm{уменьшить}$ загрузку. Данный алгоритм реализован в созданном прототипе аппаратуры Первичного триггера электромагнитного калориметра. Работа прототипа аппаратуры с предложенным алгоритмом была проверена стендовыми измерениями и экспериментами на пучке электронов. Полученные результаты удовлетворяют ключевому требованию калориметра - энергетическое разрешение в режиме реального времени лучше $5 \%$ для энергии сигнального электрона.

Ключевые слова: процессы с нарушением лептонного аромата в мюонных распадах, система предварительного отбора событий (Первичный триггер), калориметр, эксперимент СОМЕТ, распад мюона в связанном состоянии с ядром

Для цитирования. Система предварительного отбора событий электромагнитного калориметра эксперимента СОМЕТ / Д. В. Шёлковый [и др.] // Вес. Нац. акад. навук Беларусі. Сер. фіз.-мат. навук. - 2019. - Т. 55, № 1. - С. $97-109$. https://doi.org/10.29235/1561-2430-2019-55-1-97-109

\author{
Dz. V. Shoukavy', D. N. Grigoriev ${ }^{2,3,4}$, L.B. Epshteyn ${ }^{2,3,4}$, Yu. V. Yudin ${ }^{2,4}$ \\ ${ }^{1}$ B. I. Stepanov Institute of Physics of the National Academy of Sciences of Belarus, Minsk, Belarus \\ ${ }^{2}$ Budker Institute of Nuclear Physics of Siberian Branch Russian Academy of Sciences, Novosibirsk, Russia \\ ${ }^{3}$ Novosibirsk State Technical University, Novosibirsk, Russia \\ ${ }^{4}$ Novosibirsk State University, Novosibirsk, Russia
}

\title{
ELECTROMAGNETIC CALORIMETER OF THE TRIGGER SYSTEM FOR THE COMET EXPERIMENT
}

Abstract. In this paper, on the basis of the Monte-Carlo simulation results a signal processing algorithm for determination of the energy deposited in real time by incident particles has been developed and implemented in the created electronics prototype of the trigger system for an electromagnetic calorimeter of the COMET experiment. The energy thresholds for trigger cells are determined which make it possible to select signal events - an electron with a momentum of $105 \mathrm{MeV} / \mathrm{c}$, and significantly reduce a rate of background events. The electronics prototype of the trigger system has been verified by testbench measurements and electron beam experiments. The obtained results satisfy the key requirement of the calorimeter the energy resolution in real time is better than $5 \%$ for the signal electron energy.

Keywords: charged lepton flavour violation process, trigger system, calorimeter, COMET experiment, muon decay in orbit

For citation. Shoukavy Dz. V., Grigoriev D. N., Epshteyn L. B., Yudin Yu. V. Electromagnetic calorimeter of the trigger system for the COMET experiment. Vestsi Natsyianal'nai akademii navuk Belarusi. Seryia fizika-matematychnykh navuk= Proceedings of the National Academy of Sciences of Belarus. Physics and Mathematics series, 2019, vol. 55, no. 1, pp. 97-109 (in Russian). https://doi.org/10.29235/1561-2430-2019-55-1-97-109

Введение. Стандартная модель (СМ) - это единая теория, описывающая электрослабое и сильное взаимодействие элементарных частиц, в основе которой лежит принцип локальной калибровочной инвариантности, т. е. инвариантности уравнений движения по отношению к произвольным преобразованиям координат пространства-времени [1]. СМ позволяет с хорошей точностью описать большинство полученных на сегодняшний день экспериментальных данных в физике элементарных частиц. Однако, несмотря на существенный прорыв, связанный с экспериментальным 
обнаружением на Большом адронном коллайдере последней недостающей частицы CM - бозона Хиггса $[2,3]$, Стандартную модель нельзя считать полностью завершенной схемой, так как ряд фундаментальных вопросов по-прежнему остается открытым. Во-первых, в рамках СМ нейтрино с самого начала предполагаются безмассовыми; но, как стало очевидно после 1998 г., у них есть небольшие массы покоя, и нейтрино разных поколений могут, смешиваясь, осциллировать, т. е. превращаться друг в друга [4]. Во-вторых, в рамках СМ нет полностью удовлетворительных кандидатов на роль темной материи и темной энергии, плотность которых, согласно физическим и астрофизическим данным, составляет примерно 23 и 73 \% от плотности массы всей Вселенной соответственно. Стандартная модель не может дать объяснения наблюдаемой асимметрии вещества и антивещества, а также в ней отсутствует гравитационное взаимодействие. Таким образом, несмотря на все успехи Стандартной модели, актуальным остается вопрос о создании единой калибровочной теории, отвечающей на поставленные фундаментальные вопросы и описывающей все известные в природе взаимодействия.

Для преодоления внутренних проблем Стандартной модели был предложен целый ряд ее расширений: суперсимметричные теории, теории великого объединения, модели с дополнительными измерениями пространства-времени и др. Теории, выходящие за рамки СМ, называют физикой за пределами Стандартной модели, или Новой физикой, а поиск явлений и процессов, выходящих за рамки СM, представляет собой одну из главных задач современной физики. Поиск Новой физики возможен как в высокоэнергетичных экспериментах, таких как ATLAS и CMS на Большом адронном коллайдере, так и в прецизионных, - осуществляемых при относительно низких энергиях, но с высокой интенсивностью пучков. При этом оба класса экспериментов дают взаимно дополняющую информацию.

Поиск процессов с нарушением лептонного аромата в мюонных распадах. Эксперимент СОМЕТ. В физике для классификации частиц используется лептонное число (лептонный заряд) квантовое число, которое сопоставляется каждому поколению лептонов. Лептонам трех разных поколений сопоставляются лептонные квантовые числа (лептонные ароматы) Le, $\mathrm{L} \mu, \mathrm{L} \tau$ - электронный, мюонный и таонный ароматы соответственно. Частицы, не являющиеся лептонами, обладают нулевыми лептонными числами. В настоящее время все экспериментальные данные свидетельствуют о том, что во всех процессах, происходящих в замкнутой системе в результате сильных, электромагнитных и слабых взаимодействий, каждое лептонное число $\mathrm{Le}, \mathrm{L} \mu$ и $\mathrm{L} \tau \mathrm{co}-$ храняется независимо. Однако нет серьезных теоретических предпосылок для их строгого сохранения, так как сохранение лептонных ароматов не обусловлено никакой глобальной калибровочной симметрией. Более того, механизмы их нарушения возникают естественным образом практически во всех теориях за рамками СМ. Стоит отметить, что явление осцилляции нейтрино можно объяснить в рамках Стандартной модели, сделав ее незначительную модификацию путем введения массы нейтрино. Однако даже в этом случае процессы с нарушением лептонного аромата хотя формально не запрещены, но экстремально сильно подавлены, и вероятность таких распадов составляет меньше $1 \cdot 10^{-50}$ из-за малости массы нейтрино. Следовательно, обнаружение процесса, протекающего с нарушением лептонного числа, станет явным свидетельством физики за рамками СМ.

Несмотря на множество теоретических предсказаний существования переходов, сопровождающихся нарушением закона сохранения лептонного квантового числа $(\mu \rightarrow \mathrm{e} \gamma, \mu \rightarrow$ eеe, $\tau \rightarrow$ е $\gamma / \mu \gamma, \tau \rightarrow 3$ лептона, когерентный переход $\mu \rightarrow$ е в поле атомного ядра и др.), поиск таких процессов в различных экспериментах в пределах их чувствительности пока не увенчался успехом [5-8]. На рис. 1 приведена временная зависимость повышения чувствительности существующих и планируемых экспериментов по поиску процессов, проходящих с нарушением закона сохранения лептонного аромата в мюонных распадах.

Проект COMET (от англ. COherent Muon to Electron Transition) [9] является одним из прецизионных экспериментов, исследующих расширенную теорию физики элементарных частиц. Главная его задача - поиск когерентной конверсии мюона в электрон в области ядра без излучения нейтрино: $\mu^{-}+N(A, Z) \rightarrow \mathrm{e}^{-}+N(A, Z)$ ( $\mu-$ е конверсия), с чувствительностью одного события $\sim 2,6 \cdot 10^{-17}$, которая превосходит результаты лучшего предыдущего эксперимента SINDRUM-2 [5] на 4 по- 


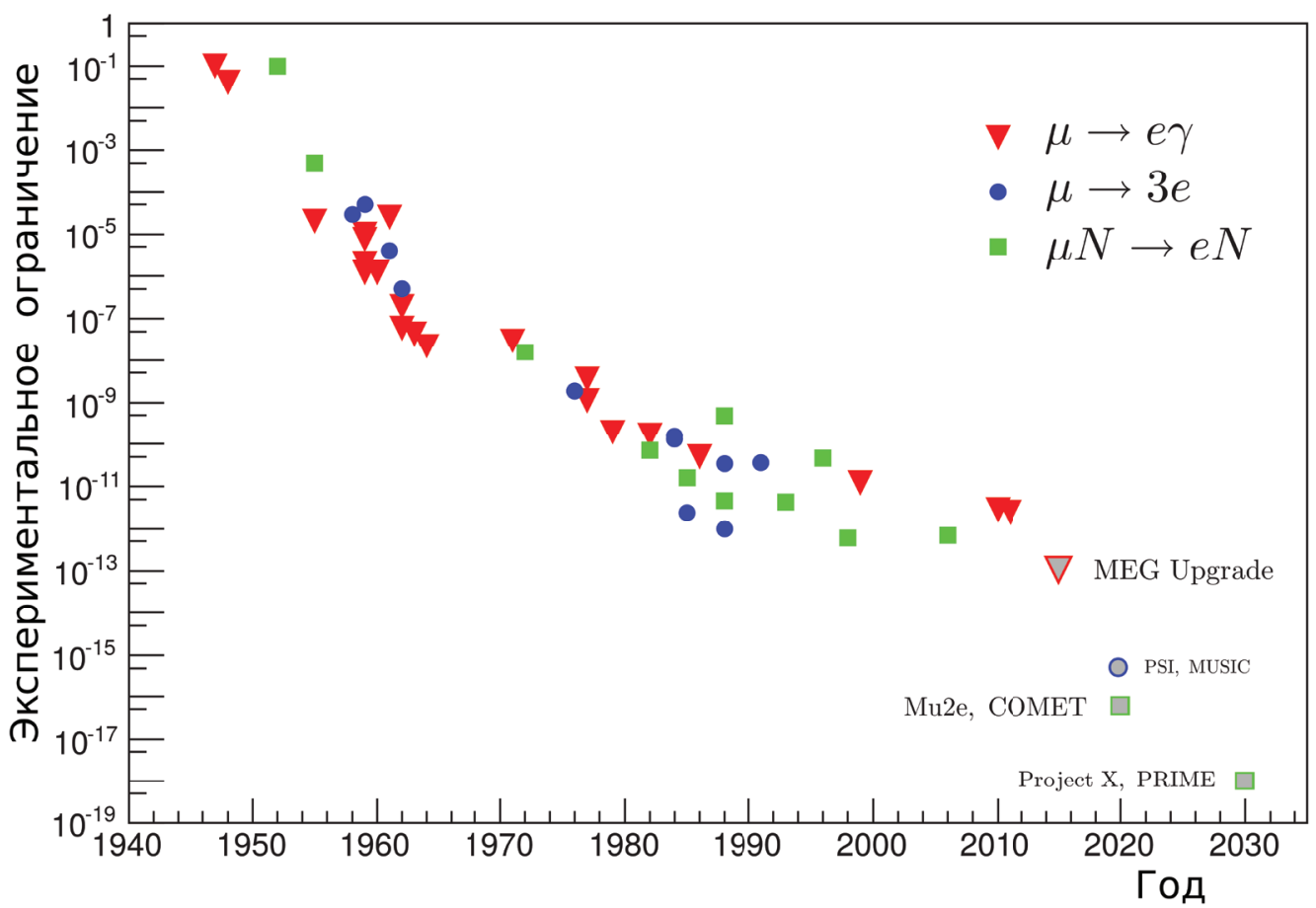

Рис. 1. История повышения чувствительности существующих и планируемых экспериментов по поиску процессов, проходящих с нарушением закона сохранения лептонного аромата в мюонных распадах

Fig. 1. Time evolution of the sensitivity increase of the existing and prospective experiments searching for the processes with lepton flavour conservation violation in muon decays

рядка. Такой переход нарушает закон сохранения числа лептонных ароматов на одну единицу, при этом общее количество лептонов не изменяется. Эксперимент СОМЕТ проводится на ускорителе протонов с рекордной интенсивностью на ускорительном комплексе J-PARC (Япония). В связи со сложностью эксперимента принято решение разделить его на 2 этапа. На первом будет использоваться только одна С-образная транспортная система, соответствующая постепенному повороту транспортируемых частиц на $90^{\circ}$. Физическая задача этого этапа - достичь чувствительности $3 \cdot 10^{-15}$ с детектором на основе цилиндрической дрейфовой камеры. Вторая цель изучение состава мюонного пучка и измерение фонов, что необходимо для разработки второго этапа, так как указанные данные отсутствуют. Эти измерения будут проведены с помощью детекторной секции, состоящей из пяти станций координатного детектора на основе дрейфовых трубок (строу-трекер), и электромагнитного калориметра на базе сцинтилляционных кристаллов; эта секция является прототипом для второго этапа эксперимента.

На втором этапе для достижения заявленной чувствительности будет использоваться полномасштабная транспортная линия соленоидов с поворотом на $360^{\circ}$, в результате чего улучшается точность отбора частиц по электрическому заряду и импульсу. Строу-трекер должен точно измерять импульс частицы по отклонению в магнитном поле и давать информацию о пространственных координатах частицы. Дополнительно он позволяет идентифицировать частицы с помощью измерения удельных потерь энергии $(d E / d x)$ в связке с калориметром, который будет предоставлять информацию об отношении энергии кластера в калориметре, ассоциированного с треком, к импульсу этого трека $(E / p)$.

Электромагнитный калориметр. Электромагнитный калориметр - это часть детектора, основанная на сцинтилляционных кристаллах LYSO [6]. Он покрывает область радиусом 50 см и расположен после координатной системы по направлению движения частиц. Основные задачи калориметра заключаются в выработке сигнала запуска для сигнальных событий и подавлении запусков от фоновых событий, а также в измерении энергии электронов. Для обеспечения допустимой частоты запусков от фоновых событий энергетическое разрешение калориметра должно быть лучше 5 \% при энергии 105 МэВ. Калориметр также должен обеспечивать пространственное 
разрешение лучше $1 \mathrm{~cm}$. Чтобы исключить ошибки измерений из-за наложений сигналов при высоких пиковых загрузках, аппаратура калориметра должна обнаруживать наложение сигналов от частиц, пришедших с интервалом по времени 220 нс и менее, и исключать такие события из измерений.

Высокая сегментация требуется для уменьшения вклада от наложения фоновых событий наложения, а также для обеспечения достаточного пространственного разрешения при поиске треков. Калориметр состоит из кристаллов сечением $2 \times 2 \mathrm{~cm}^{2}$ и длинной $12 \mathrm{~cm}$, что соответствует 10,5 радиационных длин. Для покрытия области детектора радиусом 50 см необходимо 1920 кристаллов LYSO. Основным конструктивным элементом калориметра являются модули, состоящие их четырех $(2 \times 2)$ кристаллов. Для улучшения светосбора каждый кристалл модуля обернут в два слоя фольгой из алитированного майлара, а модуль - в фольгу из тефлона. К каждому кристаллу подсоединяется лавинный фотодиод.

Система предварительного отбора событий. Система предварительного отбора событий (СПОС), или Первичный триггер (ПТ), электромагнитного калориметра должна удовлетворять следующим основным требованиям: высокая эффективность к сигнальным событиям, энергетическое разрешение не ниже 5 \%, чтобы обеспечить допустимую частоту запусков для записи событий от частиц с энергией в пределах выбранного диапазона порогов.

Напомним, что $\mu-$ е конверсия является двухчастичным процессом, в котором энергия сигнальных электронов фиксирована:

$$
E_{\mathrm{e}}=m_{\mu}-B_{\mu}-E_{\text {отд }}
$$

где $m_{\mu}$ - масса мюона, $B_{\mu}$ - энергия связи мезоатома в состоянии $1 s, E_{\text {отд }}$ - энергия отдачи ядра. Последняя значительно меньше массы мюона $m_{\mu}$, поэтому энергия вылетевшего электрона зависит от материала, в котором остановится мюон, например в случае алюминия (материал мишени, останавливающей мюоны в эксперименте СОМЕТ) $E_{\mathrm{e}}=104,97$ МэВ, для титана $-E_{\mathrm{e}}=104,3$ МэВ.

Для достижения заявленной чувствительности к единичному событию необходимо получение интенсивного пучка мюонов, в частности в условиях первого этапа эксперимента COMET требуется $1 \cdot 10^{9}$ остановившихся мюонов в мишени в секунду. Мюоны, захваченные атомами алюминия, имеют вероятность $39,1 \%$ распасться по каналу $\mu^{-} \rightarrow e^{-}+v_{\mu}+\bar{v}_{e}$. Большинство таких распадов дают высокоэнергетические электроны, вылетающие из мишени. Поскольку эти электроны происходят от остановок мюонов, их распределение по времени совпадает с распределением по времени потенциального конверсионного электрона. В результате, в условиях сверхвысокой интенсивности остановок мюонов в мишени, распад мюона в связанном состоянии с ядром (Decay in Orbit - DIO) является основным источником фона при поиске процесса $\mu-$ конверсии. Следовательно, СПОС должна эффективно отсеивать эти фоновые события с целью снижения интенсивности потока экспериментальных данных до приемлемого уровня, сохраняя при этом высокую эффективность к полезным событиям на уровне не ниже 90 \%. Детальное теоретическое описание энергетического спектра электронов от DIO-распада мюонов, захваченных в алюминии, приведено в [10] и показано на рис. 2.

Спектр DIO-электронов вблизи сигнальной энергии электрона от $\mu-\mathrm{e}$ конверсии, изображенный на рис. 2, $b$, дает объяснение, почему требуется высокое энергетическое разрешение калориметра, так как он должен подтвердить измерения импульса сигнального электрона от строу-трекера, который обладает более высоким разрешением по импульсу.

Работа ПТ основана на простом алгоритме, который запускает триггер в случае превышения величины энергетического порога на энерговыделение в кластере. Согласно рис. 2, a пик энергетического распределения электронов от DIO-распада мюонов, захваченных в алюминии, находится в районе 46 МэВ, поэтому приходим к необходимости создать триггер с высокой пороговой энергией - в идеальном случае выше 80 МэВ (см. рис $3, b$ ). В этом случае Первичный триггер будет пропускать небольшую долю DIO-событий. Варьируя величину порога на запуск ПТ, необходимо обеспечить компромисс между загрузкой триггера, приемлемой для записи на диск, и его высокой эффективностью к полезным событиям. 

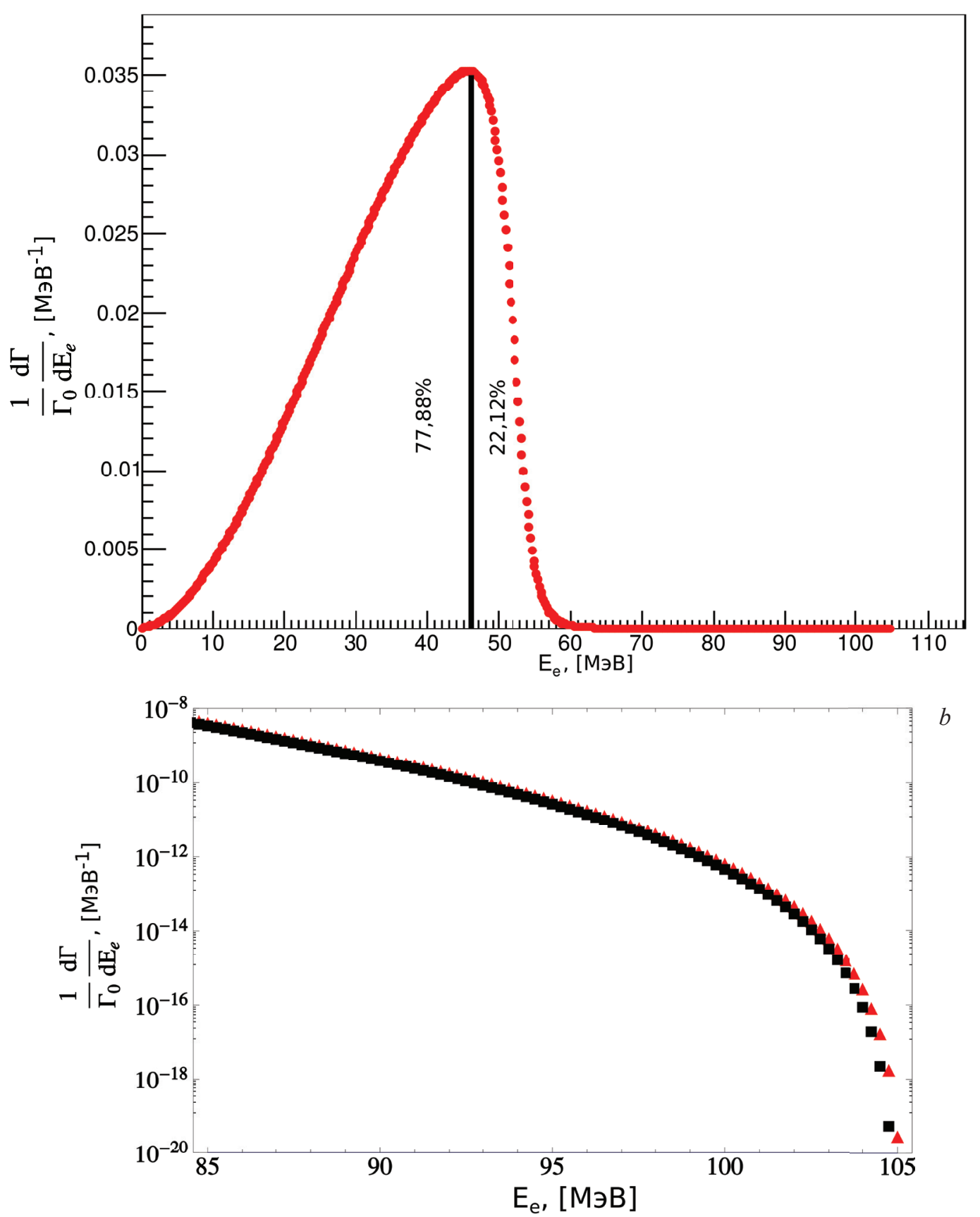

Рис. 2. Энергетический спектр электронов от распада мюонов, захваченных в алюминии (a), прямая линия соответствует значению энергии 46 МэВ; энергетический спектр электронов вблизи конечной точки (б), квадраты соответствуют спектру, учитывающему поправку на отдачу ядра, треугольники - спектру, пренебрегающему отдачей

Fig. 2. Electron energy spectrum in muon decay in orbit for aluminum (a), the straight line corresponds to an energy value of $46 \mathrm{MeV}$; endpoint region of the electron spectrum for aluminum (b), the squares correspond to the spectrum with recoil effects, triangles show the spectrum neglecting recoil

При энергиях около 100 МэВ электромагнитный ливень, вызванный попадающей частицей, будет распределяться по многим кристаллам калориметра. Для восстановления полной энергии входящих в калориметр частиц необходимо суммировать энерговыделение по нескольким кристаллам, смежным с кристаллом с максимальным энерговыделением. С целью уменьшения объема информации, обрабатываемой в системе запуска, в предварительной электронике производится аналоговое суммирование сигналов с кристаллов каждого модуля $2 \times 2$, и суммарный сигнал является базовой единицей (триггерной ячейкой). Для энергетической реконструкции в режиме реального времени доступны только сигналы триггерных ячеек, поэтому важно выбрать 
оптимальное количество триггерных ячеек, которые должны быть отнесены к одному кластеру для суммирования энергии, удовлетворяющему вышеперечисленным требованиям. Для построения оптимального алгоритма кластеризации было проведено моделирование методом МонтеКарло, при этом сравнивалось три размера кластера:

$-1 \times 1$ триггерных ячеек, соответствующий $2 \times 2$ кристаллам (1 модуль калориметра);

$-2 \times 2$ триггерных ячеек, соответствующий $4 \times 4$ кристаллам (4 модуля калориметра);

$-3 \times 3$ триггерных ячеек, соответствующий $6 \times 6$ кристаллам (9 модулей калориметра).

В случае с кластером $1 \times 1$ триггерная ячейка с максимальным энерговыделением принималась за кластер. Для случая с размером кластера $2 \times 2$ он искался следующим образом: сначала происходило суммирование по всем возможным комбинациям триггерных ячеек размером $2 \times 2$. Затем искалась максимальная триггерная сумма, которая в итоге и принималась за сработавший кластер. В случае с размером кластера $3 \times 3$ его поиск осуществлялся аналогично случаю $2 \times 2$ с единственной разницей в том, что суммирование происходило по всем возможным комбинациям $3 \times 3$ триггерных ячеек.

Моделирование было выполнено с использованием официального инструментария эксперимента COMET - ICEDUST (Integrated Comet Experimental Data User Software Toolkit) [11]. Был сгенерирован 1 млн электронных событий из мюонной стоп-мишени с импульсом $105 \mathrm{MэB} / \mathrm{c}$ и изотропным распределением направления движения. На рис. 3 показано распределение энерговыделения для каждого размера кластера.

Также проводилось моделирование эффективности запуска в зависимости от порога, налагаемого на восстановленную энергию для каждого размера кластера (рис. 4). Эффективность триггера определялась как доля сигнальных событий с энергией выше выбранного порога срабатывания. Из рис. 3 и 4 следует, во-первых, что в случае размера кластера $1 \times 1$ спектр энерговыделения очень широкий, следовательно, энергетическое разрешение данного кластера будет недопустимо большим (значительно больше 5 \%). Во-вторых, 90 \% эффективности триггера для сигнальных событий соответствует порогу 46 МэВ для одиночной триггерной ячейки, 78 МэВ для кластера $2 \times 2$ триггерных ячеек и 80,5 МэВ $-3 \times 3$ триггерных ячеек. Пропускная способность системы сбора данных (ССД) эксперимента СОМЕТ накладывает ограничение на ПТ калориметра, который должен удерживать частоту запусков на уровне 1-2 кГц. На рис. 2 видно, что величина порога 46 МэВ, соответствующая 90 \% эффективности триггера на основе единичной триггерной ячейки, находится в области пика энергетического распределения электронов от DIO-распада мюонов в алюминии, следовательно, ПТ будет иметь большую фоновую загрузку, превышающую допустимую. Использование кластера из $2 \times 2$ или $3 \times 3$ триггерных ячеек позволяет ограничить скорость потока до требуемого уровня 1-2 кГц, так как в случае 90\%-ной эффективности триггерной системы пороговая энергия находится в области логарифмического «Хвоста».

Для количественного подтверждения проведено моделирование отклика Первичного триггера на поток DIO-электронов, сгенерированных согласно энергетическому распределению (см. рис. 2), с целью оценки коэффициента подавления их потока в зависимости от эффективности триггера. Результаты моделирования для кластера $2 \times 2$ триггерных ячеек и одиночной триггерной ячейки приведены на рис. 5.

Как следует из рис. 5 , при 90\%-ной эффективности Первичного триггера на основе единичной триггерной ячейки поток DIO-электронов уменьшается только в 50 раз и значительно превосходит общую пропускную способность системы сбора данных - 10 кГц. В то же время при 90-93\%-ной эффективности ПТ на основе кластера $2 \times 2$ триггерных ячеек поток DIO-электронов уменьшается на 6 порядков, и ожидаемый поток составляет менее 1 кГц, т. е. обеспечивается нужный компромисс между загрузкой триггера и его высокой эффективностью к сигнальным событиям.

Исходя из вышесказанного, кластер размера $1 \times 1$ не может использоваться для системы предварительного отбора событий. В [12] проведено детальное сравнение работы СПОС на основе кластеров, состоящих из $2 \times 2$ и $3 \times 3$ триггерных ячеек. Шумы электроники были смоделированы следующим образом: в каждом событии и для каждого кристалла генерировалось случайное 


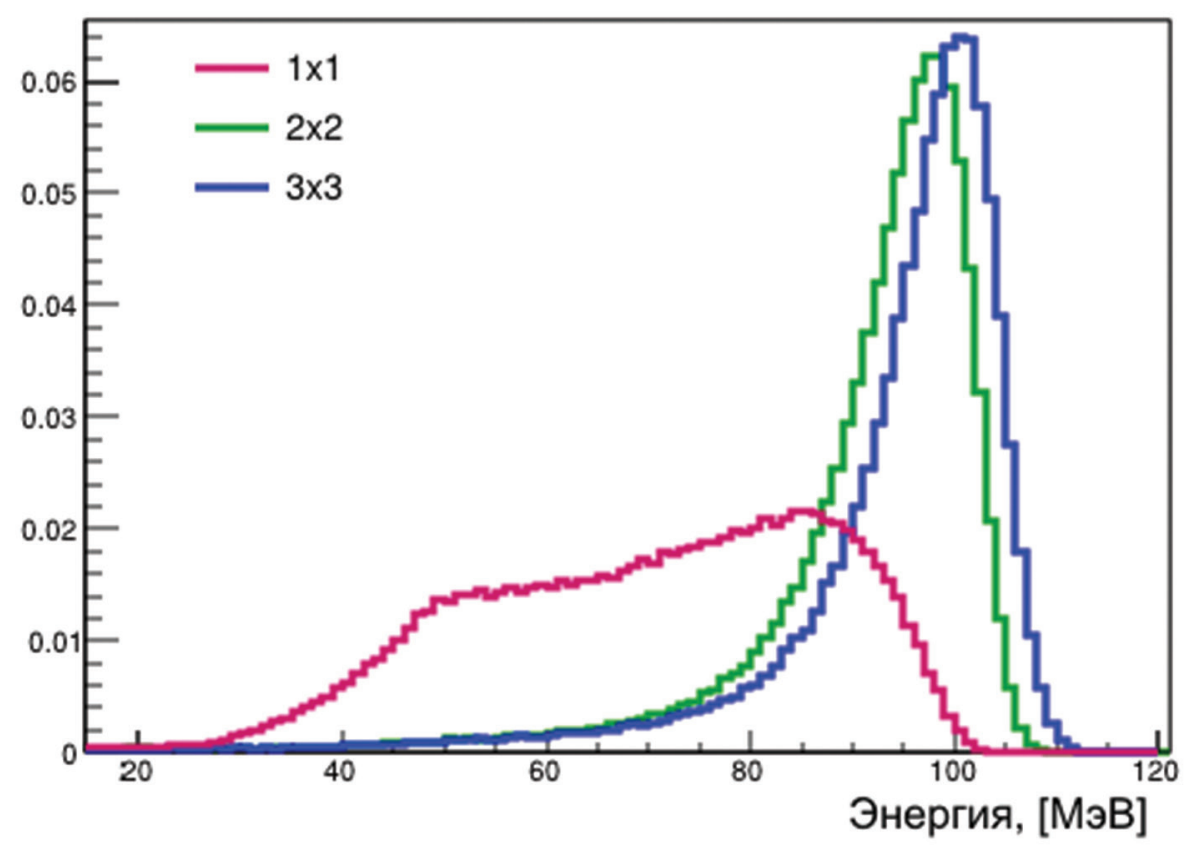

Рис. 3. Спектр энерговыделения в кластерах трех разных размеров

Fig. 3. Spectra of the deposited energy in clusters of 3 sizes

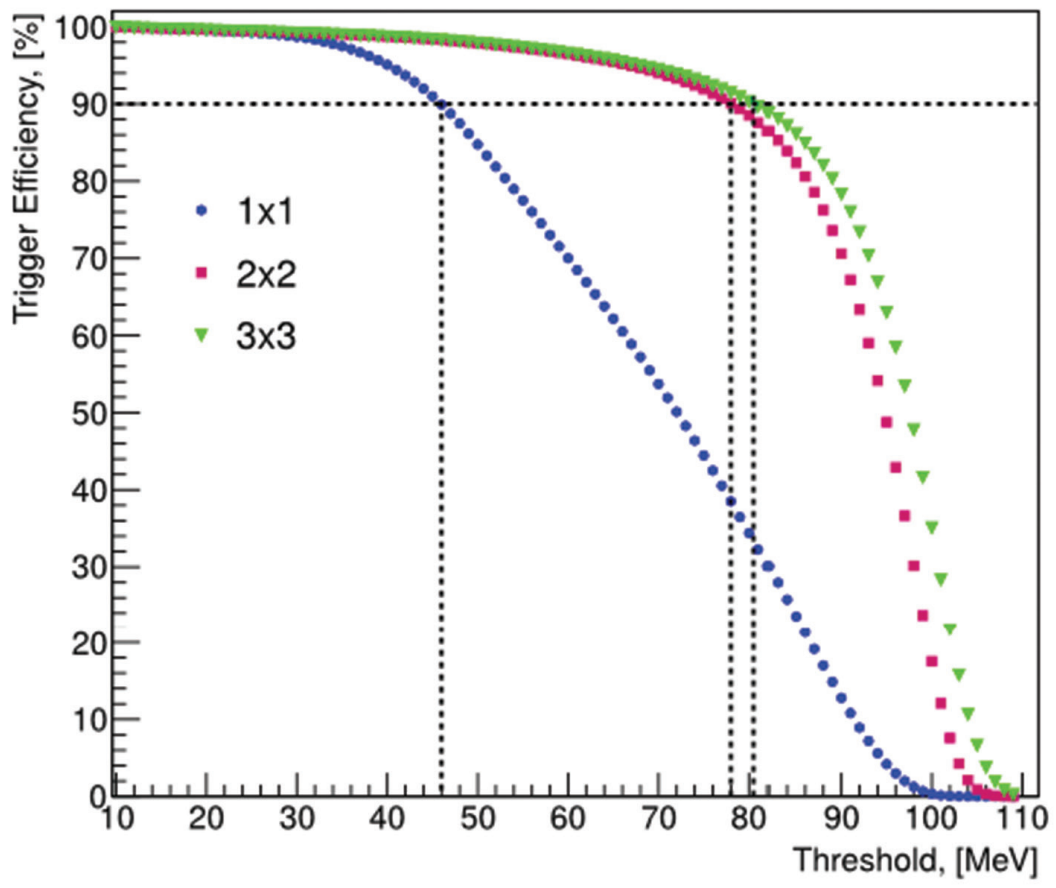

Рис. 4. Поведение эффективности запуска в зависимости от порога

Fig. 4. The behavior of the trigger efficiency as a function of the threshold

значение шума (размытое нормальным распределением с заранее заданным $\sigma_{\text {шума }}$ ), которое добавлялось к значению энерговыделения в этом кристалле. Полученные значения собранных в модуле кристаллов суммировались, что давало величину «измеренной» энергии соответствующей триггерной ячейки в этом сигнальном событии. Энергетическое разрешение для кластеров $2 \times 2$ и $3 \times 3$ оценивалось путем подбора соответствующего спектра с логарифмически нормальным распределением. Показано, что для низких уровней шума в кристалле кластер $3 \times 3$ обеспечивает лучшее энергетическое разрешение, чем кластер $2 \times 2$. Для уровней шума в кристалле от 0,6 до 0,8 МэВ оба кластера обеспечивают почти такое же энергетическое разрешение. 

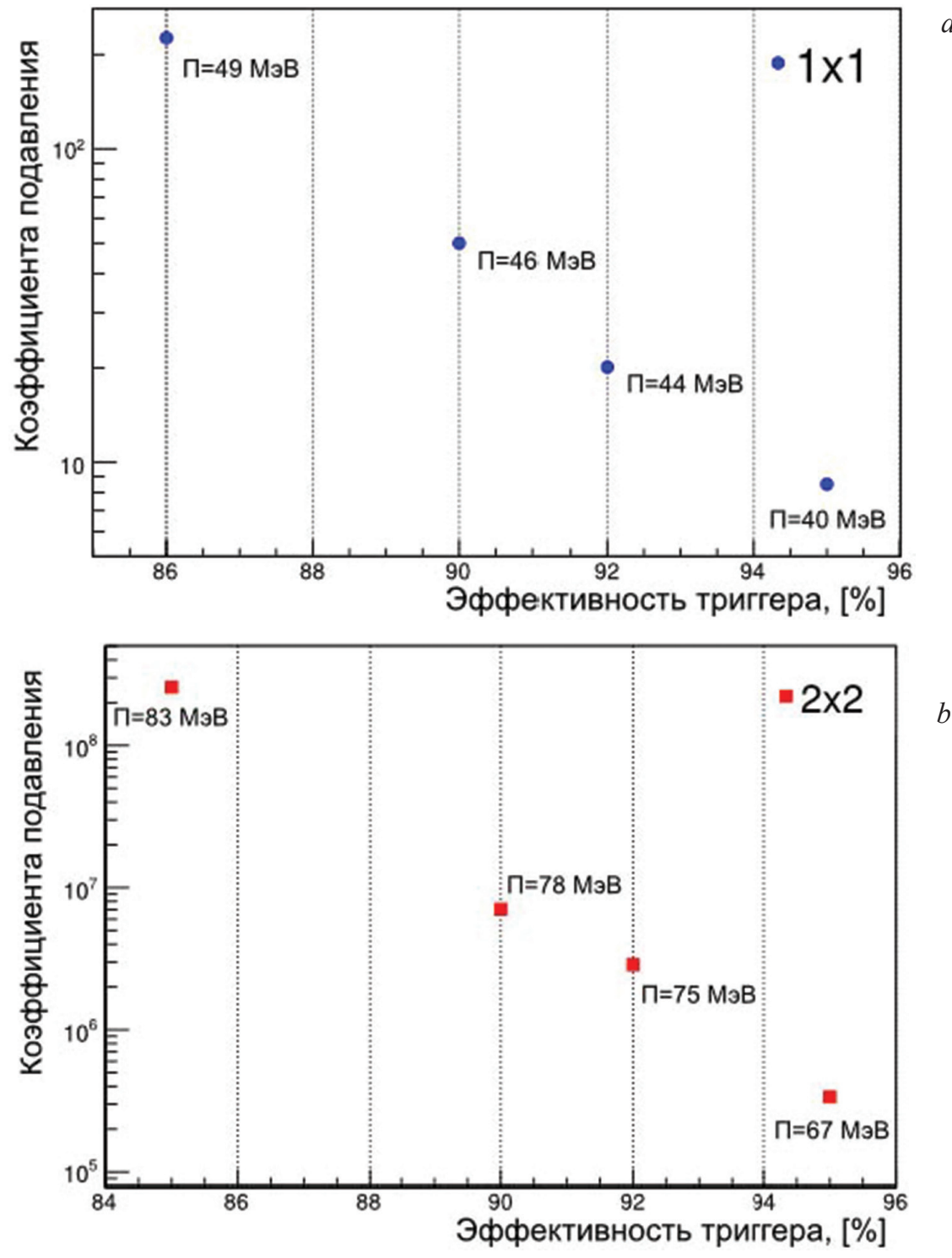

Рис. 5. Поведение коэффициента подавления потока DIO-электронов в зависимости от эффективности триггера: $a$ - одиночная триггерная ячейка; $b$ - кластер из $2 \times 2$ триггерных ячеек; П - величина пороговой энергии, соответствующая данной эффективности триггера

Fig. 5. The behavior of the DIO rejection factor as a function of the trigger efficiency for

(a) single trigger cells, (b) $2 \times 2$ trigger cells. $\Pi-$ the energy threshold corresponds to a given trigger efficiency

Для уровней шума 1 МэВ и выше кластер 2 × 2 обеспечивает лучшее энергетическое разрешение, поскольку он собирает шумы меньшего количества каналов, чем самый большой кластер.

При обработке экспериментальных данных по тестированию прототипа калориметра, состоящего из 64 кристаллов, на пучке электронов было найдено, что величина шума на кристалл составляет $\sigma_{\text {шума }}=1,15$ МэВ. Поэтому было принято решение использовать в Первичном триггере калориметра СОМЕТ размер кластера $2 \times 2$ триггерных ячеек и описанный выше алгоритм поиска оптимального кластера.

Отклик Первичного триггера на мюоны и пионы. В условиях первой стадии эксперимента COMET до калориметра будет долетать пучок, состоящий из разных частиц - преимущественно мюонов и пионов - в широком диапазоне импульсов. Поэтому важно изучить отклик триггерной системы на эти частицы с целью оценки величины порогов на триггерные ячейки для подавления фоновых событий. Было смоделировано по 1 млн мюонных и пионных событий с различными значениями импульсов: $\mu^{-}-30,40,50,60 \mathrm{MэB/c;} \pi^{-}-40,50,60 \mathrm{MэB/c.} \mathrm{Частицы}$ с более высоким импульсом не моделировались, так как они будут удалены при помощи колли- 


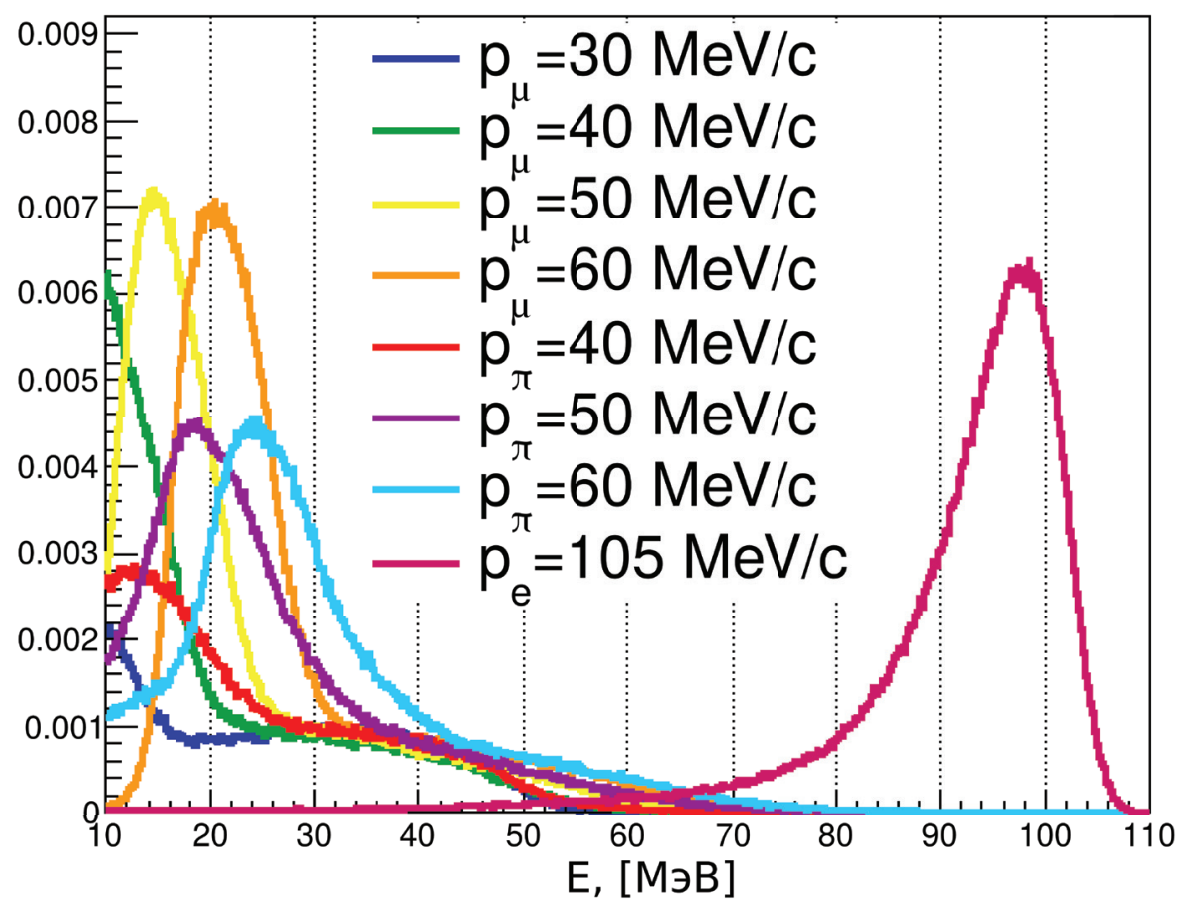

Рис. 6. Спектр энерговыделения в кластере из $2 \times 2$ триггерных ячеек Fig. 6. Spectra of the deposited energy in the cluster of $2 \times 2$ trigger cells

матора и не попадут в калориметр. Частицы с более низкими импульсами также имеют очень маленькую вероятность достичь калориметра, по этой причине пионы с импульсом $30 \mathrm{MэB/c}$ не моделировались. Спектры выделенной энергии в кластере из $2 \times 2$ триггерных ячеек для рассматриваемых частиц и сигнального события представлен на рис. 6. Следует отметить, что прежде чем наложить спектры на одну гистограмму, их предварительно нормировали на единицу. В предыдущем разделе было показано, что $90 \%$ эффективности ПТ на основе кластера из $2 \times 2$ триггерных ячеек соответствует порогу в 78 МэВ, следовательно, такой триггер будет эффективно отсеивать пионы и мюоны с рассмотренными импульсами (см. рис. 4 и 6). Например, для пиона с импульсом $60 \mathrm{MэB/c} \mathrm{отношение} \mathrm{площади} \mathrm{под} \mathrm{кривой} \mathrm{распределения} \mathrm{в} \mathrm{интервале} \mathrm{от} 78$ до 110 МэВ к полной площади составляет $100 \% \cdot S(78-110$ МэВ $) / S=0,56 \%$, в случае $\mu^{-}$с импульсом $60 \mathrm{MэВ/c-0,27 \% .}$

В связи с ограничением в 64 бита на передачу информации с ПТ калориметра обязательно должна быть передана информация о «ключевом игроке» - энерговыделение в максимальной группе и ее номер для каждого события. Следовательно, необходимо знать величину пороговой энергии на единичную триггерную ячейку с максимальным энерговыделением для уменьшения загрузки калориметра. По аналогии с предыдущей гистограммой, выбирая 90\%-ную эффективность отбора к сигнальному событию для максимальной триггерной ячейки (см. рис. 4), получаем, что доля отобранных фоновых событий значительно снижается, в частности для $60 \mathrm{MэB/c} \pi^{-}-$ $100 \% \cdot S(46-105 \mathrm{MэB}) / S=7,8 \%$, для $60 \mathrm{MэВ/c} \mu^{-}-5,5 \%$ (рис. 7 ).

Электроника Первичного триггера калориметра СОМЕТ. Для экспериментальной проверки предложенного алгоритма обработки сигналов был разработан и изготовлен прототип электроники ПТ.

Для облегчения технического обслуживания и ремонта было принято решение аналоговую часть и оцифровку сигналов триггерных ячеек вынести в отдельные оцифровывающие платы малого размера, а обработку оцифрованных данных производить на материнской плате. Каждая такая оцифровывающая плата содержит два 4-канальных 8-битных АЦП (аналого-цифровой преобразователь) с частотой дискретизации 80 МГц. Всего в материнскую плату может быть установлено до 16 оцифровывающих плат. 


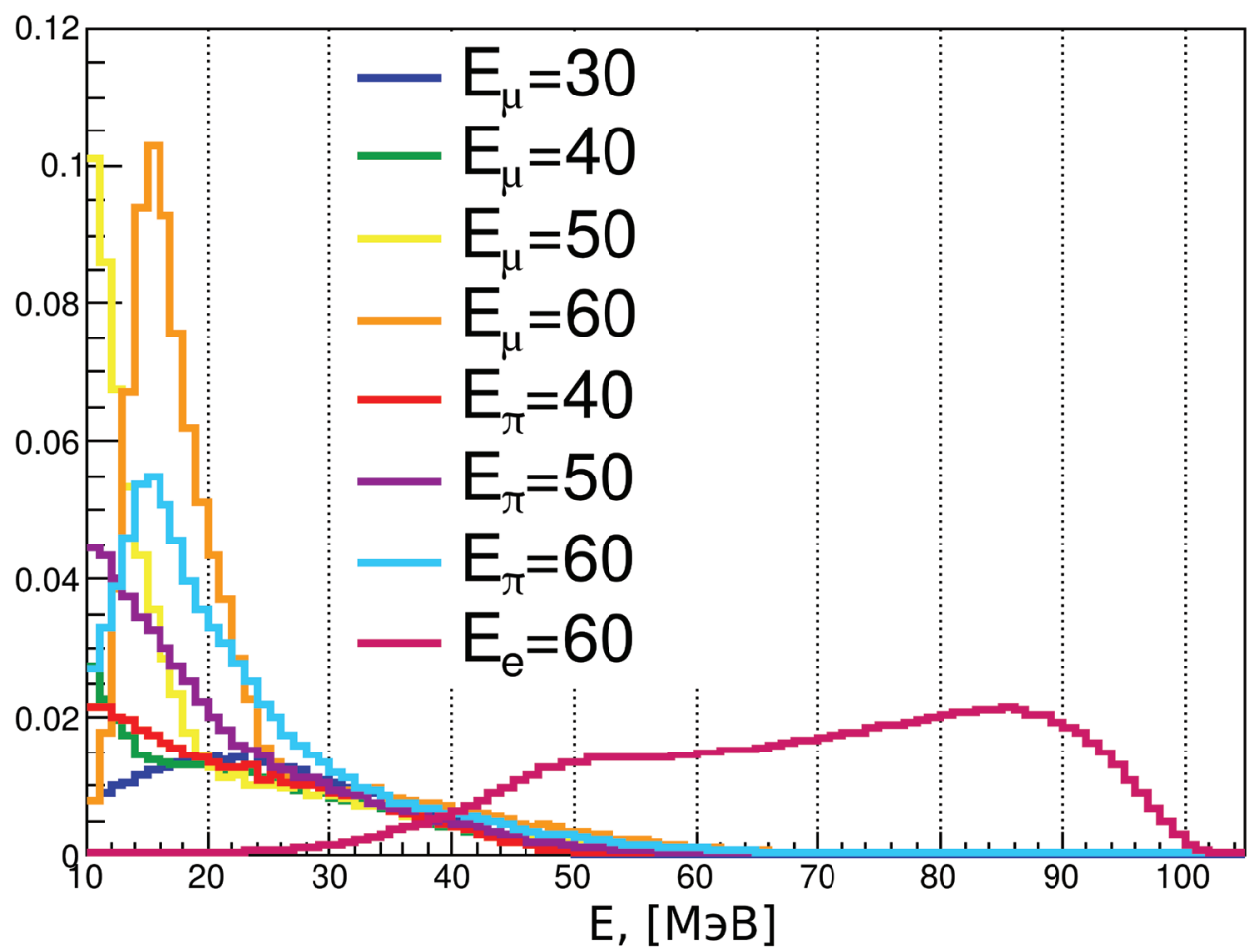

Рис. 7. Спектр энерговыделения в максимальной триггерной ячейке

Fig. 7. Spectra of the deposited energy in single trigger cells with maximum energy deposition

Все поступающие в материнскую плату Первичного триггера оцифрованные сигналы с триггерных ячеек обрабатываются разработанным алгоритмом в реальном времени в программируемой логической интегральной схеме.

Тестирование прототипа электроники на стенде. Работа прототипа электроники с запрограммированным алгоритмом была опробована с использованием нескольких комбинаций тестового сигнала от генератора таким образом, чтобы смоделировать разные варианты энерговы-

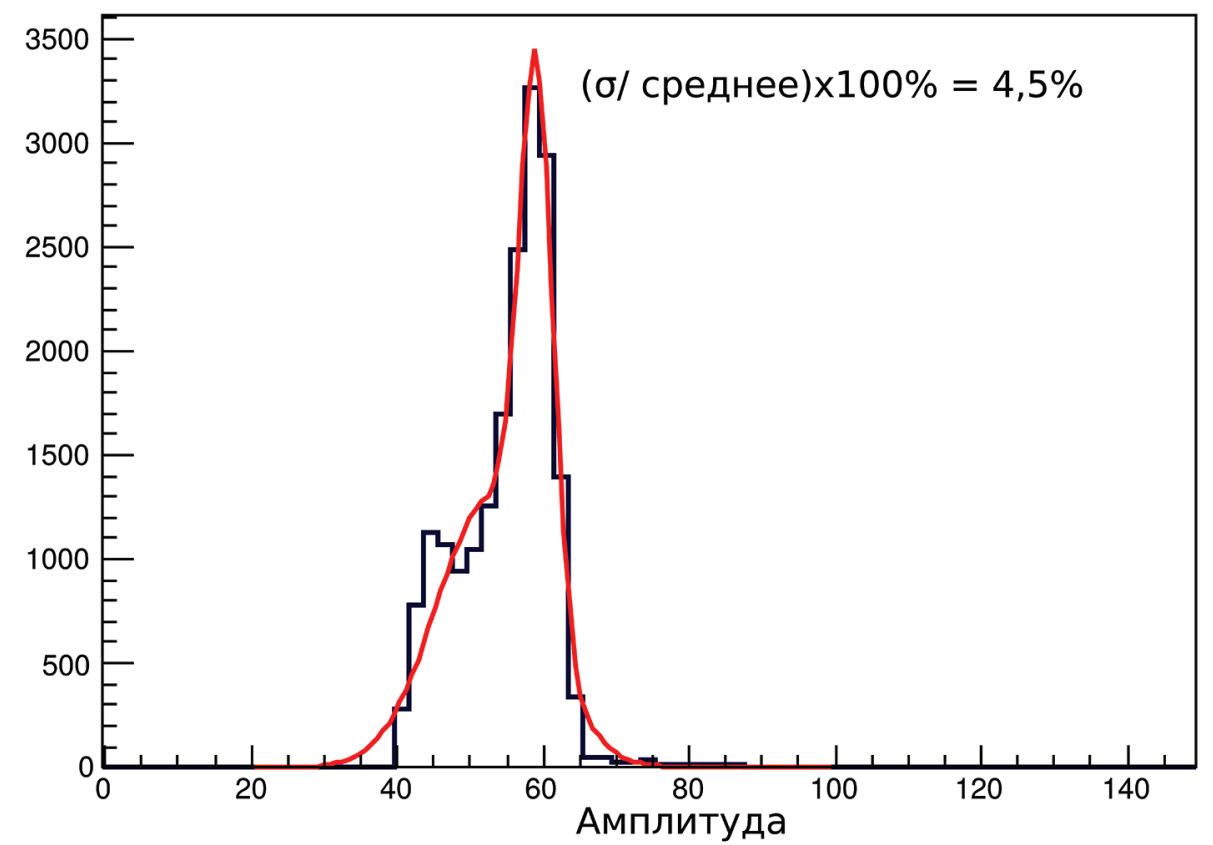

Рис. 8. Распределение измеренных амплитуд в каналах АЦП

Fig. 8. The distribution of the measured amplitudes in ADC channels 


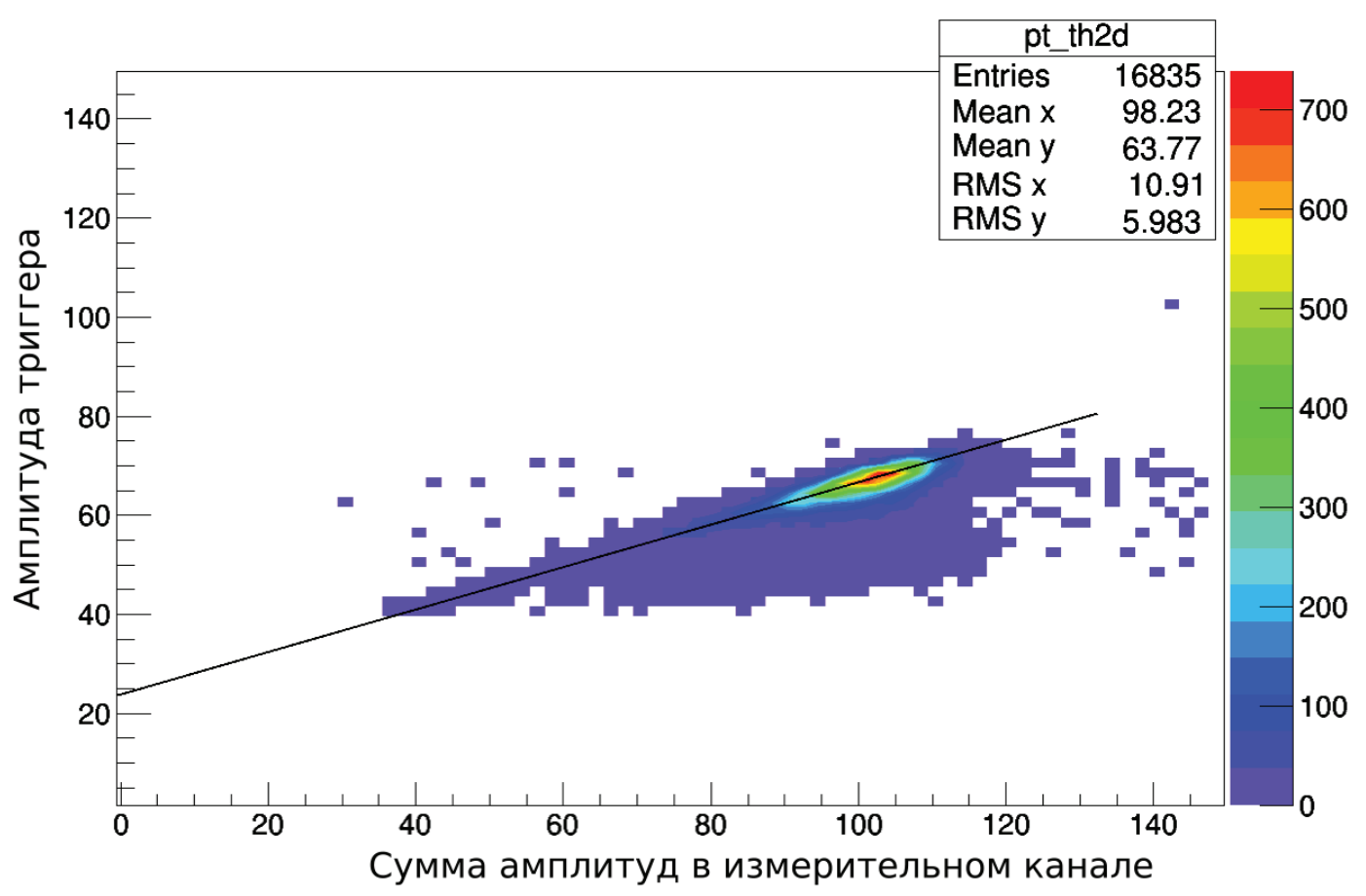

Рис. 9. График линейности каналов триггерной системы

Fig. 9. Graph of linearity of the channels of the trigger system

деления в нескольких триггерных ячейках. Для тестирования использовались прототип электроники Первичного триггера электромагнитного калориметра и одна плата предварительной электроники.

Было подтверждено, что алгоритм работает правильно: он обнаруживает сигналы ячеек вырабатывает сигнал превышения порога, находит их пиковые значения, вычисляет сумму амплитуд по каждой триггерной группе $(2 \times 2$ триггерные ячейки) и находит триггерную группу с максимальной суммой амплитуды.

Триггерные данные, в которые входят номер триггерной группы с наибольшим энерговыделением и величина энерговыделения в этой группе, вырабатывались через 0,8-1,0 мкс от момента появления сигнала от генератора (в зависимости от амплитуды сигнала).

Тестирование на пучке электронов. Работа системы также была протестирована на выведенном пучке электронов (г. Сендай, Япония). Для этого использовался прототип калориметра, состоящий из 64 кристаллов.

Было набрано 10 заходов (5 с запусками от триггерной системы калориметра и 5 - от внешней системы для измерения эффективности) около 20 тыс. событий каждый. Общая эффективность при энергии выше 30 МэВ составила 99,4 \%, более 40 МэВ - 99,7 \%. Эффективность вычислялась из соотношения количеств событий, зарегистрированных внешней системой, и событий, зарегистрированных триггерной системой калориметра. Энергетическое разрешение триггерной системы по записанным заходам получилось порядка 4,5 \% (см. рис. 8) и определялось как отношение $\sigma$ распределения к среднему значению распределения за вычетом пьедестала (рис. 9).

Заключение. Алгоритм обработки сигналов разработан, оптимизирован с помощью моделирования методом Монте-Карло и реализован в электронике ПТ калориметра. Работа алгоритма протестирована на стенде и в реальных событиях. Полученные результаты показали, что алгоритм работает корректно. Основываясь на результатах измерений, было принято решение произвести небольшие модификации в электронике, а также в будущем выполнить тестирование электроники Первичного триггера калориметра с системой глобального триггера детектора для принятия финального решения по использованию данной системы в эксперименте COMET. 
Благодарности. Работа выполнена при поддержке Белорусского республиканского фонда фундаментальных исследований (грант № Ф18Р-006) и Российского фонда фундаментальных исследований (гранты № 17-02-01073, № 18-52-00004),
Acknowledgements. The work was supported by the Belarusian Republican Foundation for Fundamental Research (grant No. Ф18P-006) and by the Russian Foundation for Basic Research (grants No. 17-02-01073, No. 18-52-00004).

\section{Список использованных источников}

1. Емельянов, В. М. Стандартная модель и ее расширения / В. М. Емельянов. - М.: Физматлит, 2007. - 584 с.

2. Observation of a new particle in the search for the Standard Model Higgs boson with the ATLAS detector at the LHC / G. Aad [et al. ATLAS Collaboration] // Phys. Lett. B. - 2012. - Vol. 716, № 1. - P. 1-29. https://doi.org/10.1016/j.physletb.2012.08.020

3. Observation of a new boson at a mass of $125 \mathrm{GeV}$ with the CMS experiment at the LHC / S. Chatrchyan [et al. CMS Collaboration] // Phys. Lett. B. - 2012. - Vol. 716, № 1. - P. 30-61. https://doi.org/10.1016/j.physletb.2012.08.021

4. Evidence for oscillation of atmospheric neutrinos / Y. Fukuda [et al. Super-Kamiokande Collaboration] // Phys. Rev. Lett. - 1998. - Vol. 81, № 8. - P. 1562-1567. https://doi.org/10.1103/PhysRevLett.81.1562

5. New Limit on the Lepton-Flavor-Violating Decay $\mu+\rightarrow \mathrm{e}+\gamma /$ J. Adam [et al. MEG Collaboration] // Phys. Rev. Lett. 2011. - Vol. 107, № 17. - P. 171801 (5p.). https://doi.org/10.1103/PhysRevLett.107.171801

6. Search for the decay $\mu+\rightarrow \mathrm{e}+\mathrm{e}+\mathrm{e}-\mathrm{ee} / \mathrm{W}$. Bertl [et al. SINDRUM Collaboration] // Nucl. Phys. B. - 1985. - Vol. 260, № 1. - P. 1-31. https://doi.org/10.1016/0550-3213(85)90308-6

7. Test of lepton-flavour conservation in $\mu \rightarrow$ e conversion on titanium / C. Dohmen [et al. SINDRUM II Collaboration] // Phys. Lett. B. - 1993. - Vol. 317, № 4. - P. 631-636. https://doi.org/10.1016/0370-2693(93)91383-x

8. A search for $\mu$-e conversion in muonic gold / W. Bertl [et al.] // Eur. Phys. J. C. - 2006. - Vol. 47, № 2. - P. 337-346. https://doi.org/10.1140/epjc/s2006-02582-x

9. Kuno, Y. A search for muon-to-electron conversion at J-PARC: the COMET experiment / Y. Kuno // Prog. Theor. Exp. Phys. - 2013. - Vol. 2013, № 2. - 022C011. https://doi.org/10.1093/ptep/pts089

10. Czarnecki, A. Muon decay in orbit: Spectrum of high-energy electrons / A. Czarnecki, X. G. Tormo, W. J. Marciano // Phys. Rev. D. - 2011. - Vol. 84, № 1. - P. 013006. https://doi.org/10.1103/physrevd.84.013006

11. COMET Phase-I Technical Design Report / R. Abramishvili [et al. COMET Collaboration]. [Electronic resource]. Mode of access: http://comet.kek.jp/Documents_files/PAC-TDR-2016/COMET-TDR-2016_v2.pdf. - Date of access: 01.05.2016.

12. The level-1 trigger system for the electromagnetic calorimeter of the COMET experiment / L. B. Epshteyn [et al.] // J. Instrum. - 2017. - Vol. 12. - P. C01064 (8 p.). https://doi.org/10.1088/1748-0221/12/01/c01064

\section{References}

1. Emel'yanov V. M. Standard Model and Its Extensions. Moscow, Fizmatlit Publ., 2007. 584 p. (in Russian).

2. Aad G., Abajyan T., Abbott B., Abdallah J., Abdel Khalek S., Abdelalim A. A., Abdinov O., Aben R., Abi B., Abolins M., AbouZeid O. S., Abramowicz H. [et al. ATLAS Collaboration]. Observation of a new particle in the search for the Standard Model Higgs boson with the ATLAS detector at the LHC. Physics Letter B, 2012, vol. 716, no. 1, pp. 1-29. https://doi. org/10.1016/j.physletb.2012.08.020

3. Chatrchyan S., Khachatryan V., Sirunyan A., Tumasyan A., Adam W., Aguilo E., Bergauer T., Dragicevic M., Erö J., Fabjan C. [et al. CMS Collaboration]. Observation of a new boson at a mass of $125 \mathrm{GeV}$ with the CMS experiment at the LHC. Physics Letter B, 2012, vol. 716, no. 1, pp. 30-61. https://doi.org/10.1016/j.physletb.2012.08.021

4. Fukuda Y., Hayakawa T., Ichihara E., Inoue K, Ishihara K., Ishino H., Itow Y., Kajita T., Kameda J., Kasuga S. [et al. Super-Kamiokande Collaboration]. Evidence for oscillation of atmospheric neutrinos. Physical Review Letters, 1998, vol. 81, no. 8, pp. 1562-1567. https://doi.org/10.1103/PhysRevLett.81.1562

5. Adam J., Bai X., Baldini A. M., Baracchini E., Bemporadab C., Bocaab G., Cattaneo P. W., Cavoto G., Ceiab F., Cerri C. [et al. MEG Collaboration]. New Limit on the Lepton-Flavor-Violating Decay $\mu+\rightarrow \mathrm{e}+\gamma$. Physical Review Letters, 2011, vol. 107, no. 17, p. 171801 (5 p.). https://doi.org/10.1103/PhysRevLett.107.171801

6. Bertl W., Egli S., Eichler R., Engfer R., Felawka L., Grab Ch., Hermes E. A., Kraus N., Lordong N., Martino J., Pruys H. S., Schaaf A. v. d., Walter H. K. Search for the decay $\mu+\rightarrow \mathrm{e}+\mathrm{e}+\mathrm{e}-$. Nuclear Physics B, 1985, vol. 260, no. 1, pp. 1-31. https://doi. org/10.1016/0550-3213(85)90308-6

7. Dohmen C., Groth K.-D., Heer B., Honecker W., Otter G., Steinrücken B., Wintz P., Djordjadze V., Hofmann J., Kozlowski T., Playfer S., Bertl W., Egger J., Herold W., Krause B., Walter H. K., Engfer R., Findeisen Ch., [et al. SINDRUM II Collaboration]. Test of lepton-flavour conservation in $\mu \rightarrow$ e conversion on titanium. Physics Letter B, 1993, vol 317, no. 4, pp. 631636. https://doi.org/10.1016/0370-2693(93)91383-x

8. Bertl W., Engfer R., Hermes E. A., Kurz G., Kozlowski T., Kuth J., Otter G., Rosenbaum F., Ryskulov N. M, van der Schaaf A., Wintz P., Zychor I. A search for $\mu$-e conversion in muonic gold. European Physical Journal C, 2006, vol. 47, no. 2, pp. 337-346. https://doi.org/10.1140/epjc/s2006-02582-x

9. Kuno Y. A search for muon-to-electron conversion at J-PARC: the COMET experiment. Progress of Theoretical and Experimental Physics, 2013, vol. 2013, no. 2, 022C011. https://doi.org/10.1093/ptep/pts089

10. Czarnecki A., Tormo X. G., Marciano W. J. Muon decay in orbit: Spectrum of high-energy electrons. Physical Review D, 2011, vol. 84, no. 1, p. 013006. https://doi.org/10.1103/physrevd.84.013006 
11. Abramishvili R., Adamov G., Akhmetshin R., Anishchik V., Aoki M., Arimoto Y., Bagaturia I., Ban Y., Bondar A., Calas Y., Canfer S., Cardenas Y., Chen S. [et al. COMET Collaboration]. COMET Phase-I Technical Design Report. 2016. Available at: http://comet.kek.jp/Documents_files/PAC-TDR-2016/COMET-TDR-2016_v2.pdf

12. Epshteyn L. B., Akhmetshin R. R., Grigoriev D. N., Kazanin V. F., Melnik A. S., Shoukavy Dz. V., Yudin Yu. V. The level-1 trigger system for the electromagnetic calorimeter of the COMET experiment. Journal of Instrumentation, 2017, vol. 12, p. C01064 (8 p.). https://doi.org/10.1088/1748-0221/12/01/c01064

\section{Информация об авторах}

Шёлковый Дмитрий Викторович - кандидат физико-математических наук, ведущий научный сотрудник, Институт физики им. Б. И. Степанова Национальной академии наук Беларуси (пр. Независимости, 68-1, 220072, г. Минск, Республика Беларусь). E-mail: shoukavy@ifanbel. bas-net.by

Григорьев Дмитрий Николаевич - кандидат физико-математических наук, заведующий лабораторией, Новосибирский государственный университет (ул. Пирогова, 2, 630090, г. Новосибирск, Российская Федерация); заведующий лабораторией, Институт ядерной физики им. Г. И. Будкера СО РАН (пр. Академика Лаврентьева, 11, 630090, г. Новосибирск, Российская Федерация); доцент, Новосибирский государственный технический университет (пр. К. Маркса, 20, 630073, г. Новосибирск, Российская Федерация). E-mail: d.n.grigoriev@inp.nsk.su

Эпштейн Леонид Борисович - научный сотрудник, Новосибирский государственный университет (ул. Пирогова, 2, 630090, г. Новосибирск, Российская Федерация); научный сотрудник, Институт ядерной физики им. Г. И. Будкера СО РАН (пр. Академика Лаврентьева, 11, 630090, г. Новосибирск, Российская Федерация); старший преподаватель, Новосибирский государственный технический университет (пр. К. Маркса, 20, 630073, г. Новосибирск, Российская Федерация). E-mail: 1.b.epshteyn@inp.nsk.su

Юдин Юрий Валерьевич - кандидат технических наук, старший научный сотрудник, Новосибирский государственный университет (ул. Пирогова, 2, 630090, г. Новосибирск, Российская Федерация); старший научный сотрудник, Институт ядерной физики им. Г. И. Будкера СО РАН (пр. Академика Лаврентьева, 11, 630090, г. Новосибирск, Российская Федерация). E-mail: yu.v.yudin @inp.nsk.su

\section{Information about the authors}

Dzmitry V. Shoukavy - Ph. D. (Physics and Mathematics), Leading Researcher, B. I. Stepanov Institute of Physics of the National Academy of Sciences of Belarus (68-1, Nezavisimosti Ave., 220072, Minsk, Republic of Belarus). E-mail: shoukavy@ifanbel.bas-net.by

Dmitry N. Grigoriev - Ph. D. (Physics and Mathematics), Head of the Laboratory, Novosibirsk State University (2, Pirogov Str., 630090, Novosibirsk, Russian Federation); Head of the Laboratory, 630090, Budker Institute of Nuclear Physics of the Siberian Branch of the Russian Academy of Sciences (11, Akademician Lavrentiev Ave., 630090, Novosibirsk, Russian Federation); Assistant Professor, Novosibirsk State Technical University (20, K. Marks Ave., 630073, Novosibirsk, Russian Federation). E-mail: d.n.grigoriev@inp. nsk.su

Leonid B. Epshteyn - Researcher, Novosibirsk State University (2, Pirogov Str., 630090, Novosibirsk, Russian Federation); Researcher, Budker Institute of Nuclear Physics of the Siberian Branch of the Russian Academy of Sciences (11, Akademician Lavrentiev Ave., 630090, Novosibirsk, Russian Federation); Senior Lecturer, Novosibirsk State Technical University (20, K. Marks Ave., 630073, Novosibirsk, Russian Federation). E-mail: 1.b.epshteyn@inp.nsk.su

Yurii V. Yudin - Ph. D. (Engineering), Senior Researcher, Novosibirsk State University (2, Pirogov Str., 630090, Novosibirsk, Russian Federation); Senior Researcher, Budker Institute of Nuclear Physics of the Siberian Branch of the Russian Academy of Sciences (11, Akademician Lavrentiev Ave., 630090, Novosibirsk, Russian Federation). E-mail: yu.v.yudin @inp.nsk.su 